\title{
Progressive myoclonic epilepsy type 6
}

INSERM

\section{Source}

INSERM. (1999). Orphanet: an online rare disease and orphan drug data base.

Progressive myoclonic epilepsy type 6. ORPHA:280620

A rare, genetic, neurological disorder characterized by early-onset, prog ressive ataxia associated with myoclonic seizures (frequently associated with other seizure types such as generalized tonic-clonic, absence and drop attacks), scoliosis of variable severity, areflexia, elevated creatine kinase serum levels, and relative preservation of cognitive function until late in the disease course. 